Supporting Information

Insight into Chemical Reduction and Charge Storage Mechanism of 2,2'-dipyridyl disulfide Toward Stable Lithium Organic Battery

Qianqian Fan, Yubing $\mathrm{Si}^{*}$, Wei Guo, and Yongzhu Fu*

College of Chemistry, Zhengzhou University, Zhengzhou 4500o1, P. R. China

*yfu@zzu.edu.cn

*ybsi@zzu.edu.cn

\title{
Experiment Methods
}

\section{Chemical and Materials}

Toray carbon paper (CP, Fuel Cell Earth, thickness: $190 \mu \mathrm{m}$, o\% Teflon coating), commercial liquid carbonate electrolyte (1.o $\mathrm{M} \mathrm{LiPF}_{6}$ in ethylene carbonate: diethyl carbonate $=1: 1 \mathrm{vol}$, Canrd), lithium bis(trifluoromethanesulfonimide) (LiTFSI, $\mathrm{LiN}\left(\mathrm{CF}_{3} \mathrm{SO}_{2}\right)_{2}, 99.95 \%$, SigmaAldrich), lithium nitrate $\left(\mathrm{LiNO}_{3}, 99.99 \%\right.$, Canrd), Sodium hexafluorophosphate $\left(\mathrm{NaPF}_{6}\right.$, 99.99\%, Canrd), 1,2-dimethoxyethane (DME, 99.99\%, Canrd), 1,3-dioxolane (DOL, 99.99\%, Canrd), Diethylene glycol dimethyl ether (DEGDME, 99.99\%, Canrd), phenyl disulfide (PDS, $\mathrm{C}_{12} \mathrm{H}_{10} \mathrm{~S}_{2}, 99 \%$, Sigma-Aldrich), 2,2'-dipyridyl disulfide (PyDS, $\mathrm{C}_{10} \mathrm{H}_{8} \mathrm{~N}_{2} \mathrm{~S}_{2}, 98 \%$, Tokyo Chemical Industry Co. Ltd).

\section{Preparation of lithiated and sodiated graphite carbon paper}

The lithiated graphite carbon paper ( $\mathrm{Li}-\mathrm{CP}$ ) was prepared according to the reported method. ${ }^{1}$ First, the Toray carbon paper was cut into circular discs with a diameter of $1.2 \mathrm{~cm}$ and mass of $8.9 \pm 0.1 \mathrm{mg}$ before dried in an air-oven for $24 \mathrm{~h}$ at $100{ }^{\circ} \mathrm{C}$, then assembled in $\mathrm{CR}_{2032}$ coin cells inside an Argon-filled glove box $\left(\mathrm{H}_{2} \mathrm{O}<0.1 \mathrm{ppm}, \mathrm{O}_{2}<0.1 \mathrm{ppm}\right)$. For assembling the half cells for lithiation, $30 \mu \mathrm{L}$ of liquid carbonate electrolyte was added into a Toray carbon paper electrode, followed by a Celgard 2400 separator, $20 \mu \mathrm{L}$ of additional electrolyte, and lithium metal anode. Finally, the cells were crimped for the electrochemical discharge outside the glove box. The cells were galvanostatically discharged to $0.01 \mathrm{~V}$ at $\mathrm{C} / 40$ rate $\left(1 \mathrm{C}=167 \mathrm{~mA} \mathrm{~g}^{-1}\right)$ on a LAND CT20o1A battery test system (Wuhan LAND electronics Co., Ltd, China) then opened inside the glove box. Finally, the lithiated graphite carbon paper was washed with DME/DOL (1:1 v/v) mixture solvent thoroughly to remove all soluble species, e.g., lithium salt $\mathrm{LiPF}_{6}$ and carbonate solvent, for characterization and use in the Li/organosulfide cells containing lithiated graphite carbon paper electrodes (denoted as Li|Li-CP-organosulfide). 
The sodiated graphite carbon paper was prepared to the reported method. ${ }^{2}$ For assembling the half cells for sodiation, $30 \mu \mathrm{L}$ of $1 \mathrm{M} \mathrm{NaPF}_{6}$ dissolved in DEGDME was added into a Toray carbon paper electrode, followed by a Celgard 2400 separator, $20 \mu \mathrm{L}$ of additional electrolyte, and sodium metal anode. Finally, the cells were crimped for the electrochemical discharge outside the glove box. The cells were galvanostatically discharged to $0.01 \mathrm{~V}$ at C/40 rate on a LAND CT20o1A battery test system (Wuhan LAND electronics Co., Ltd, China) then opened inside the glove box. Finally, the sodiated graphite carbon paper was washed with DME/DOL (1:1 v/v) mixture solvent thoroughly to remove all soluble species, e.g., sodium salt $\mathrm{NaPF}_{6}$, for characterization and use in the $\mathrm{Na}$ /organosulfide cells containing sodiated graphite carbon paper electrodes (denoted as $\mathrm{Na} / \mathrm{Na}-\mathrm{CP}-$ organosulfide).

\section{Preparations of organosulfide catholytes and assembly of Li|Li-CP-organosulfide cells}

Appropriate amount of PDS or PyDS was dissolved into DOL/DME (1:1 vol) solvent or the DOL/DME solutions containing different concentrations of LiTFSI and o.15 $\mathrm{M} \mathrm{LiNO}_{3}$ to form 1 M PDS or PyDS catholyte. Li|Li-CP-organosulfide cells were assembled inside the glove box. First, $22 \mu \mathrm{L}$ of organosulfide catholyte was added into the lithiated graphite carbon paper. The theoretical capacities of PDS and PyDS in the catholyte are $1.2 \mathrm{mAh}$. Then a Celgard 2400 separator was placed on top of the electrode followed by additional blank DOL/DME (1:1 vol) solvent or electrolyte. Subsequently, lithium metal anode was placed on the separator. The cells were crimped and take out of the glove box for stabilizing over a specific period of time (days) to monitor their open circuit voltage (OCVs). The OCVs of Li|Li-CP-organosulfide cells were monitored using a LANHE battery cycler.

\section{Assembly of Li/organosulfide flow cells and electrochemical characterization}

Figure S12 shows the structure and photograph of the proof-of-concept Li/organosulfide flow cells. The electrolyte used in this experiment is $1 \mathrm{M} \mathrm{LiTFSI}$ with $0.2 \mathrm{M} \mathrm{LiNO}_{3}$ in DOL/DME. First, $16 \mu \mathrm{L}$ PDS or PyDS catholyte ( $2 \mathrm{M}$ ) was added into the CP. Then Celgard 2400 separator was placed onto the $\mathrm{CP}$ followed by $5 \mu \mathrm{L}$ blank electrolyte and lithium metal anode. The Celgard 2400 separator was moistened with $5 \mu \mathrm{L}$ blank DOL/DME solvent before assembling the cells. Subsequently, a nickel mesh was placed on the lithium anode. Two casings (bottom and top) were separated by a spacer and the assembly was conducted in the glove box. It should be noticed that the purpose of using separator is only to avoid shorting between the cathode and lithium anode. The theoretical capacity of 2.0 M organosulfides is $109 \mathrm{Ah} \mathrm{L}_{\text {catholyte }}{ }^{-1}$. The molar ratio of LiTFSI:PyDS is $1: 2$ and the concentration of $\mathrm{LiNO}_{3}$ is $0.2 \mathrm{M}$, which can sufficiently suppress the chemical reduction of PyDS by lithium anode. The mass loading of active materials was $\sim 6.2 \mathrm{mg} \mathrm{cm}^{-2}$. The effective reaction area of the cell is $1.13 \mathrm{~cm}^{2}$. Galvanostatic discharge/charge tests were performed on a LAND CT20o1A battery test system. 


\section{Material characterization}

The morphology of $\mathrm{CP}$ was investigated by a Phenom FEG scanning electron microscope (SEM). The X-ray diffraction (XRD) data of the CP, Li-CP, Li-CP-PDS, and Li-CP-PyDS electrodes after a prolonged stabilizing time (10 days) were collected on a Rigaku

MiniFlex6oo XRD Instrument equipped with $\mathrm{Cu} \mathrm{K \alpha}$ radiation in steps of $\mathrm{o.oz}^{\circ}$. The scanning rate was $0.8^{\circ} \mathrm{min}^{-1}$, and $2 \theta$ was set between $10^{\circ}$ and $70^{\circ}$.

The ${ }^{7} \mathrm{Li}$ NMR spectra were recorded at $194.4 \mathrm{MHz}$ using a Bruker AVIII50o system at room temperature. $\mathrm{LiCl}$ in $\mathrm{H}_{2} \mathrm{O}\left(1.0 \mathrm{~mol} \mathrm{~L} \mathrm{~L}^{-1}\right)$ was used as the reference (o ppm). Appropriate amounts of LiTFSI and PyDS (LiTFSI: PyDS molar ratio =1:1) were mixed in DOL/DME (1:1 vol) solvent to explore the interaction between $\mathrm{Li}^{+}$in LiTFSI and $\mathrm{N}$ atoms in PyDS.

A Waters ACQUITY UPLC I-Class PLUS liquid chromatogram coupled to a Waters Xevo G2XS QTof mass spectrometer was used to analyze the Li-CP-PDS and Li-CP-PyDS electrodes samples after a prolonged stabilizing time. The samples were immersed in $2 \mathrm{~mL}$ chromatographic methanol. $1 \mathrm{~mL}$ of this solution was taken with a pipette and added to an UPLC-MS vial with $1 \mathrm{~mL}$ additional chromatographic methanol. The column was ACOUITY UPLC BEH C 18 LC Column (2.1-10o mm, Waters). The solvent phase for UPLC was from $70 / 30 \%$ to $80 / 20 \% \mathrm{CH}_{3} \mathrm{OH} / \mathrm{H}_{2} \mathrm{O}$ between o and 4 minutes, next it was changed from $80 / 20 \%$ to $98 / 2 \% \mathrm{CH}_{3} \mathrm{OH} / \mathrm{H}_{2} \mathrm{O}$ between 4 and 9 minutes, and then it was from $98 / 2 \%$ to $70 / 30 \%$

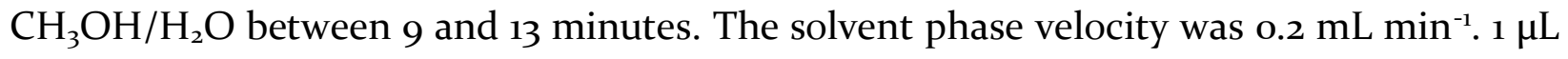
of the solution was tested in atmospheric pressure chemical ionization (APCI) positive mode.

\section{Computational details}

The density functional theory (DFT) and molecular dynamics (MD) simulations approach were employed to explore the relationships of electrolytes and disulfide molecules. To do this, the LiTFSI, $\mathrm{LiNO}_{3}$, DOL, DME, PyDS, and PhDS are firstly optimized respectively at B3LYP/Def2SVP level of theory by Gaussian o9 program.3,4 The subsequent frequency analysis was performed at the same level to verify that the structures are local minima. To evaluate the interactions of the electrolyte and disulfides, there are 3648 atoms (with 20 LiTFSI, $3 \mathrm{LiNO}_{3}, 143$ DOL, 95 DME and 10 PyDS or 10 PDS molecules included) mixed together in a $40 \times 40 \times 40 \AA^{3}$ cubic box to mimic the experimental electrolyte environment, the extended tight-binding (xTB) theoretical method was used to pre-optimize the complex system, then the 100 ps MD simulations were performed at $300 \mathrm{~K}$ to make the solvent is sufficient mixed. 5

Based on the observed experimental and MD simulations results, the coordinated PyDSLiTFSI cluster and the closet solvent molecules ( 385 atoms in total) are extracted from the snapshots and ready for the subsequent calculations. For the sake of calculation, one lithium atom $\left(\mathrm{Li}^{\circ}\right)$ was added near the PyDS ( 10.5 $\AA$ far from sulfur atom of PyDS to make sure there is no interaction initially) and then simulated by GFN-xTB approach, ${ }^{5}$ the 
Mulliken charges of the snapshots along the trajectory were calculated at B3LYP/Def2SVP level using Gaussian o9 program. ${ }^{4}$ DFT data of the charge density difference and the Mayer bond order as well as the $2 \mathrm{D}$ electrostatic potential plotting was visualized with VMD and Multiwfn programs (Figure 3). ${ }^{6,7}$ 

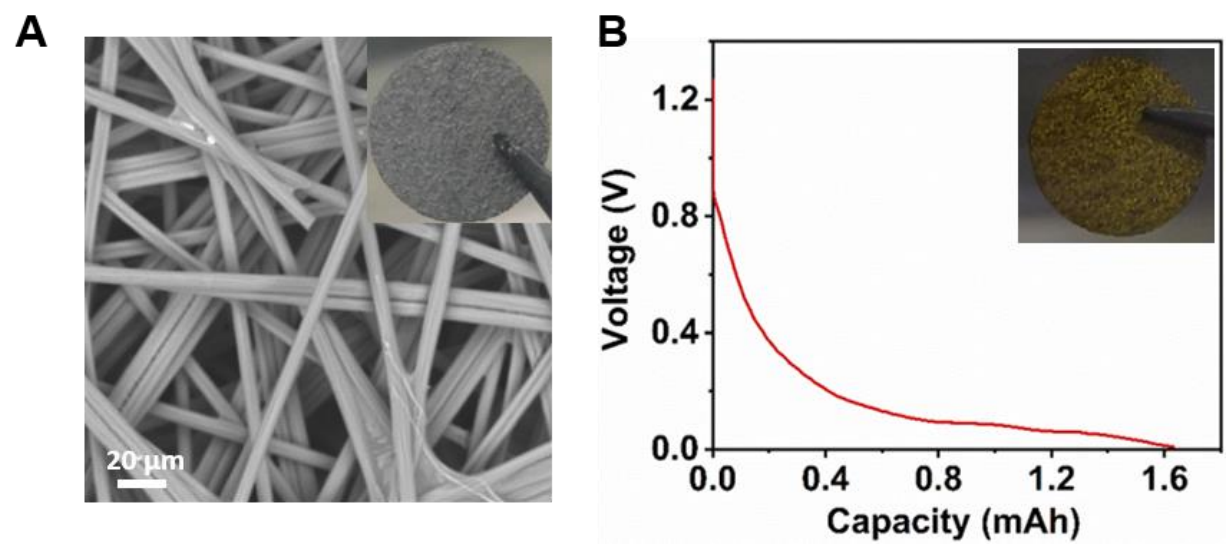

Figure S1. (A) SEM image of the woven carbon fibers of a pristine graphitic carbon paper (CP). (B) First discharge (lithiation) voltage profile of CP. The illustration is the photograph of lithiated graphitic carbon paper ( $\mathrm{Li}-\mathrm{CP})$.

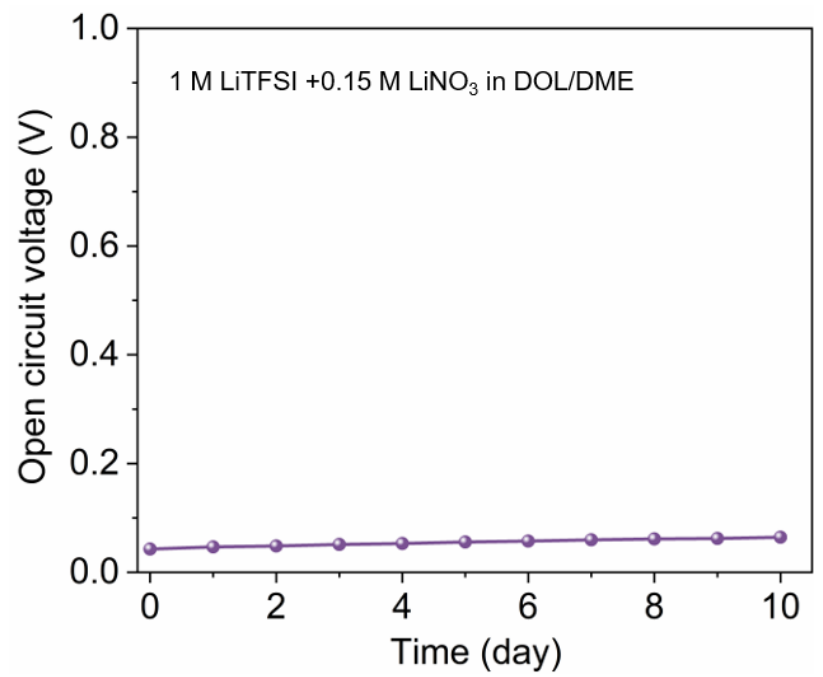

Figure S2. OCV of LilLi-CP cell in electrolyte system (1M LiTFSI with o.15 $\mathrm{M} \mathrm{LiNO}_{3}$ ) in DOL/DME). 

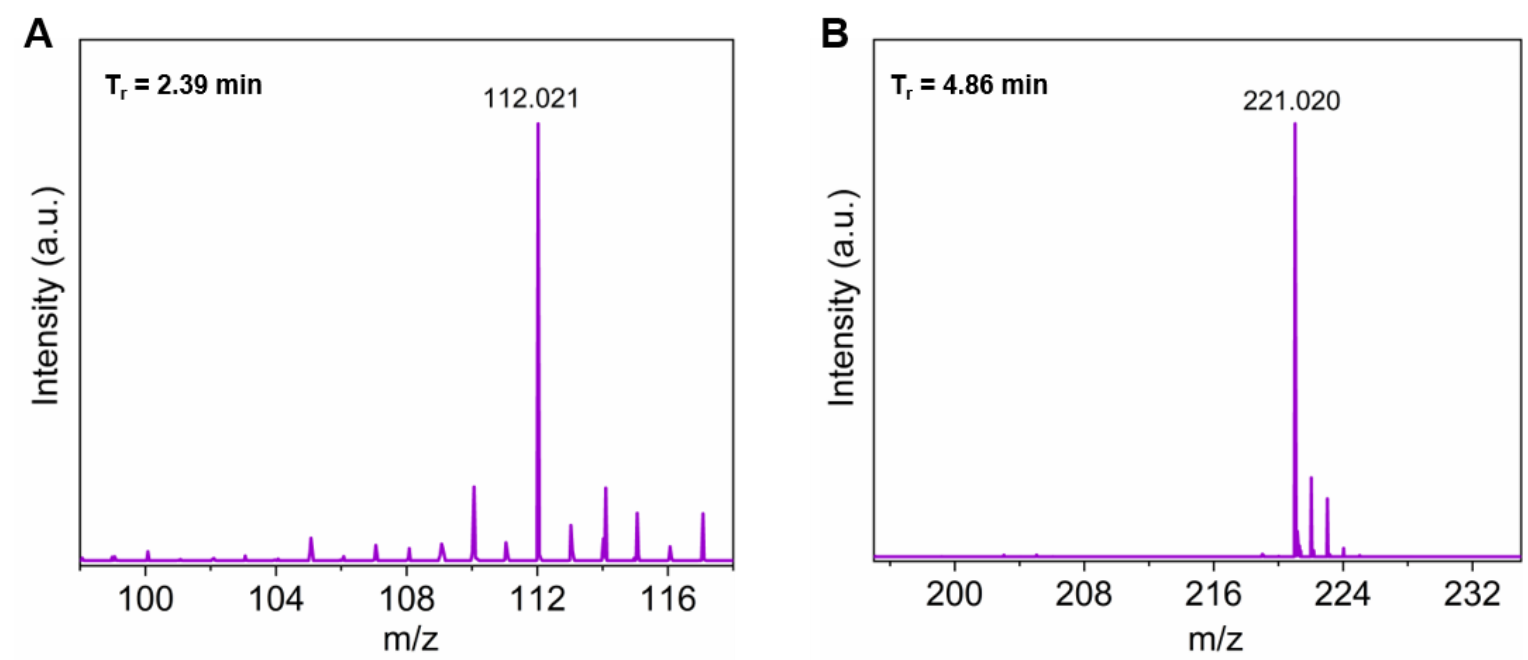

Figure S3. Corresponding MS spectra of the UV peaks of the Li-CP-PyDS electrode in etherbased electrolyte (1 M LiTFSI with $0.15 \mathrm{M} \mathrm{LiNO}_{3}$ in DOL/DME solvent) after a prolonged stabilizing time at (A) $2.39 \mathrm{~min}$ and (B) $4.86 \mathrm{~min}$.

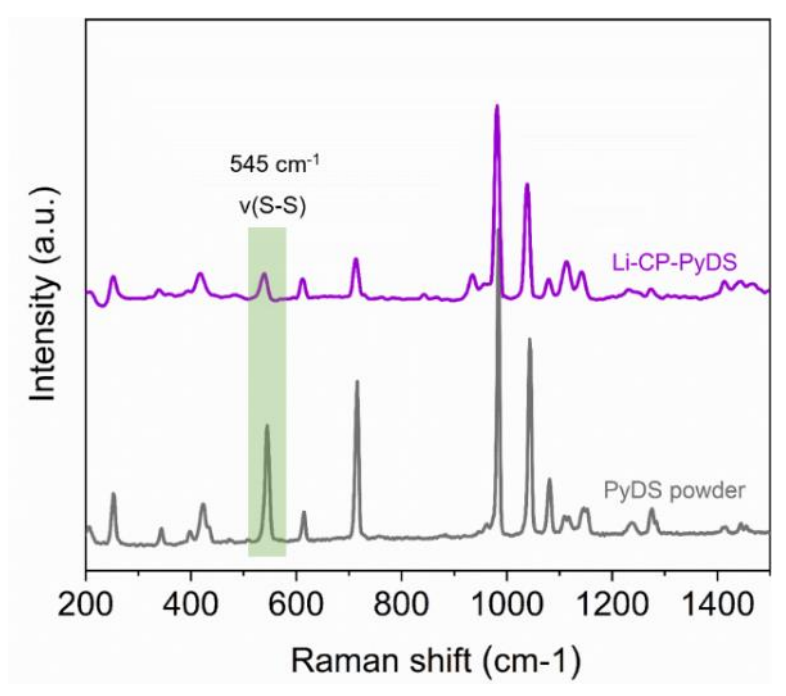

Figure S4. Raman spectra of the Li-CP-PyDS electrode in ether-based electrolyte after a prolonged stabilizing time. 

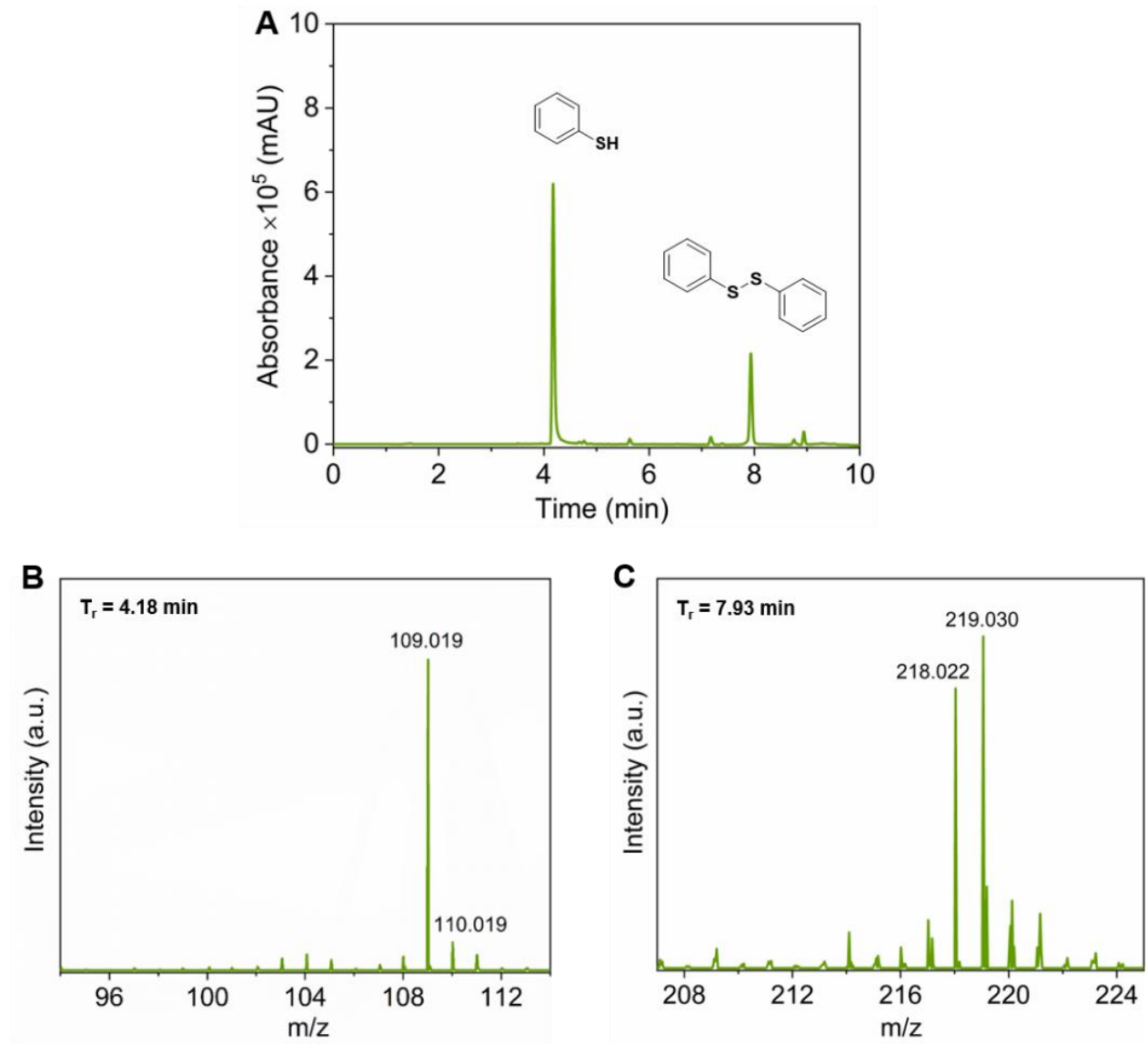

Figure S5. (A) UV absorption spectra of the Li-CP-PDS electrode in UPLC in ether-based electrolyte (1 M LiTFSI with $0.15 \mathrm{M} \mathrm{LiNO}_{3}$ in DOL/DME solvent) after a prolonged stabilizing time and the corresponding MS spectra at (B) $4.18 \mathrm{~min}$ and (C) $7.93 \mathrm{~min}$.

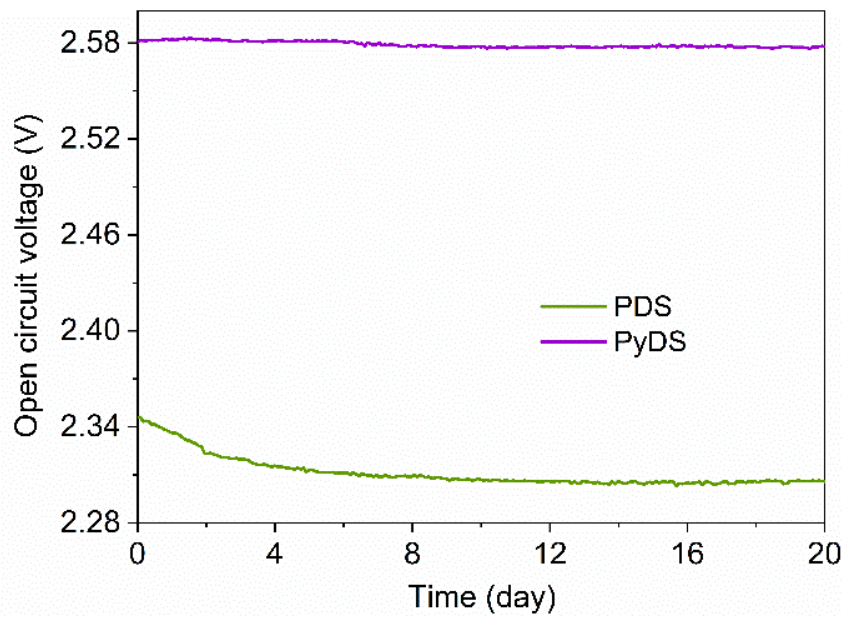

Figure S6. Self-discharge analyses of the LilPyDS (PDS) cells. The anode is lithium metal. 

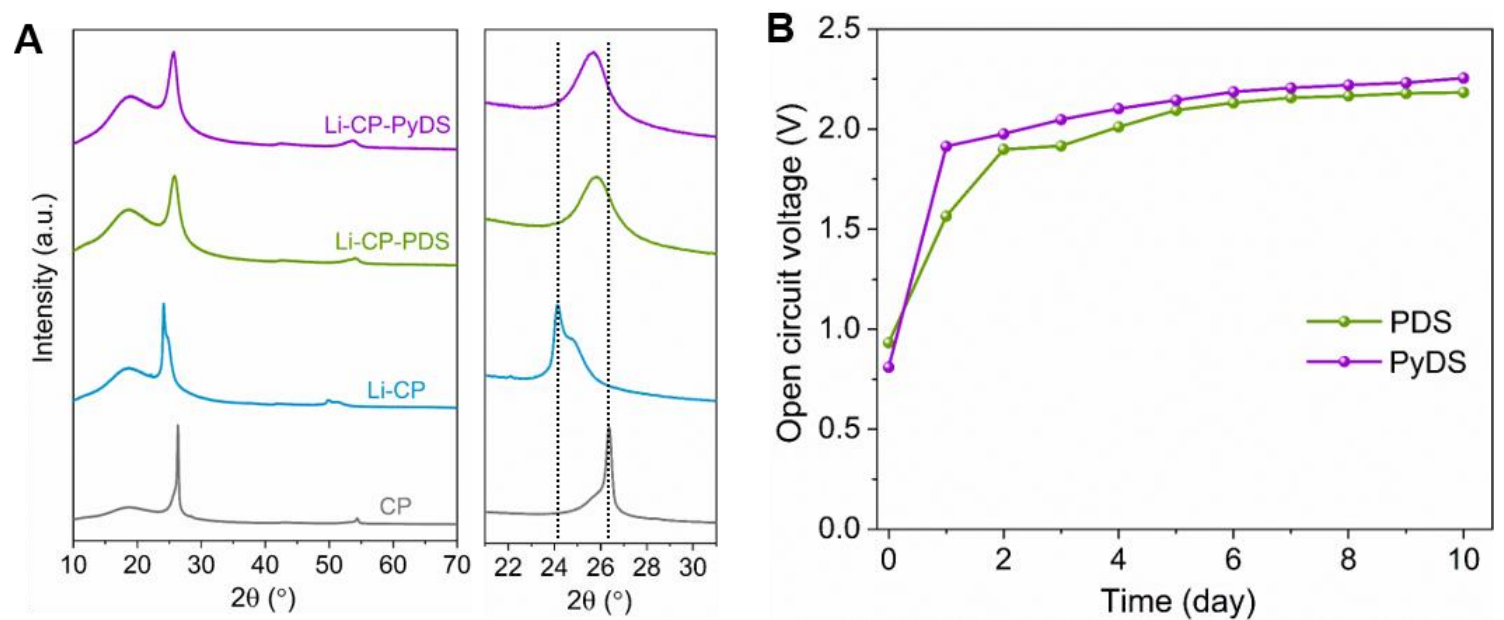

Figure S7. (A) XRD patterns of pristine CP, Li-CP, Li-CP-PDS, and Li-CP-PyDS electrodes after a prolonged stabilizing time (1o days) in pure DOL/DME solvent, with the zoomed-in XRD pattern showing the region of the (ooz) diffraction at $2 \theta=21^{\circ}-31^{\circ}$. (B) OCV of $\mathrm{Li}$ /organosulfides cells with $\mathrm{Li}-\mathrm{CP}$ electrodes as a function of stabilizing time in pure DOL/DME solvent.
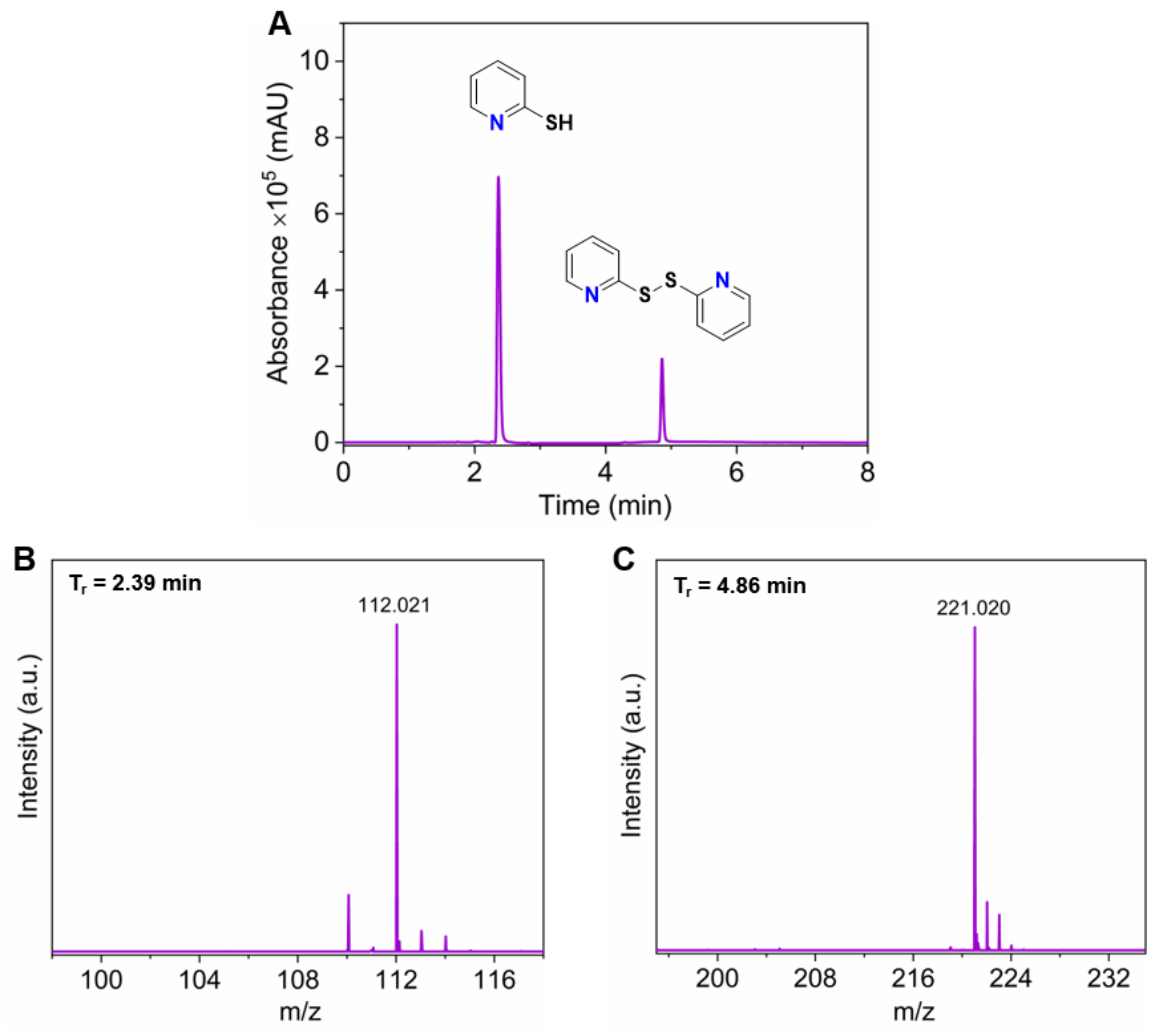

Figure S8. (A) UV absorption spectra of the Li-CP-PyDS electrode in UPLC in DOL/DME solvent after a prolonged stabilizing time and the corresponding MS spectra at (B) $2.39 \mathrm{~min}$ and (C) $4.86 \mathrm{~min}$. 


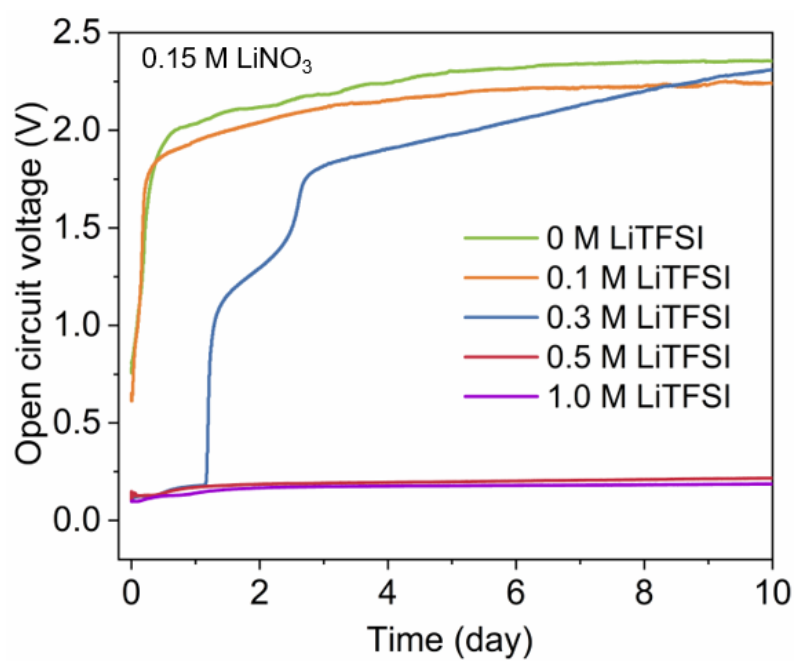

Figure S9. OCVs of the Li/PyDS cells with the Li-CP electrodes as a function of stabilizing time and with different LiTFSI concentrations and $0.15 \mathrm{M} \mathrm{LiNO}_{3}$ as electrolyte.
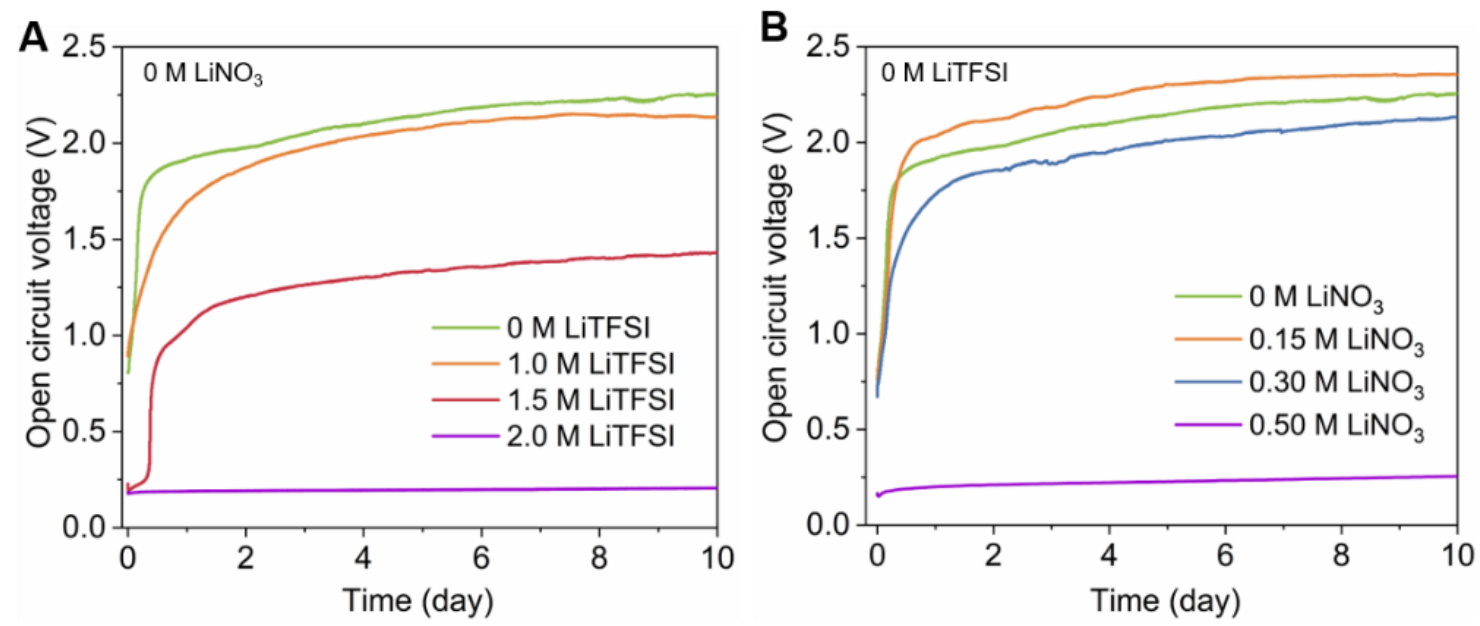

Figure S1o. OCV of Li/PyDS cells with Li-CP electrode as a function of stabilizing time (A) with different concentrations of pure LiTFSI or (B) with different concentrations of pure $\mathrm{LiNO}_{3}$ as electrolytes. 


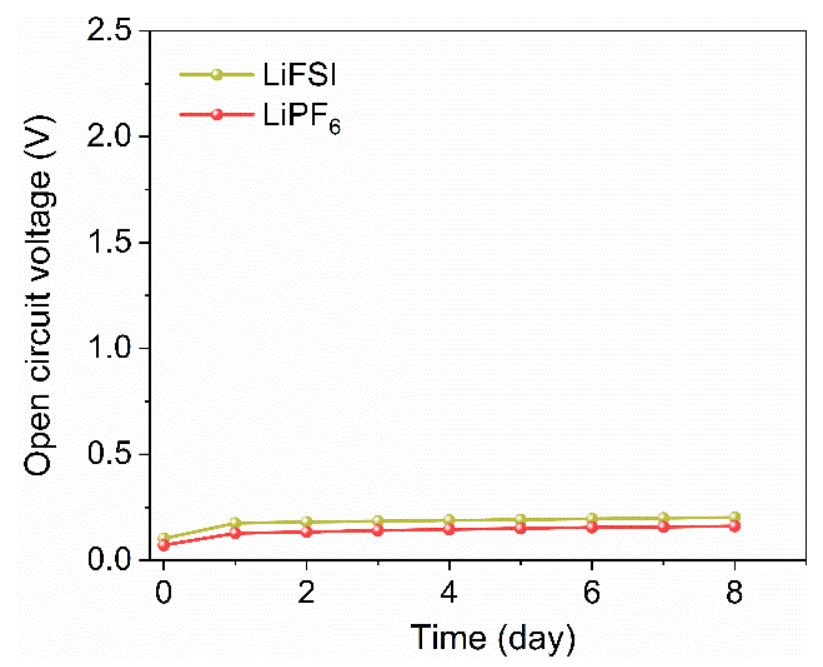

Figure S11. OCV of Li/PyDS cells with Li-CP electrode as a function of stabilizing time and with $1 \mathrm{M} \mathrm{LiFSI}$ or $1 \mathrm{M} \mathrm{LiPF}_{6}$ and $0.15 \mathrm{M} \mathrm{LiNO}_{3}$ as electrolytes.

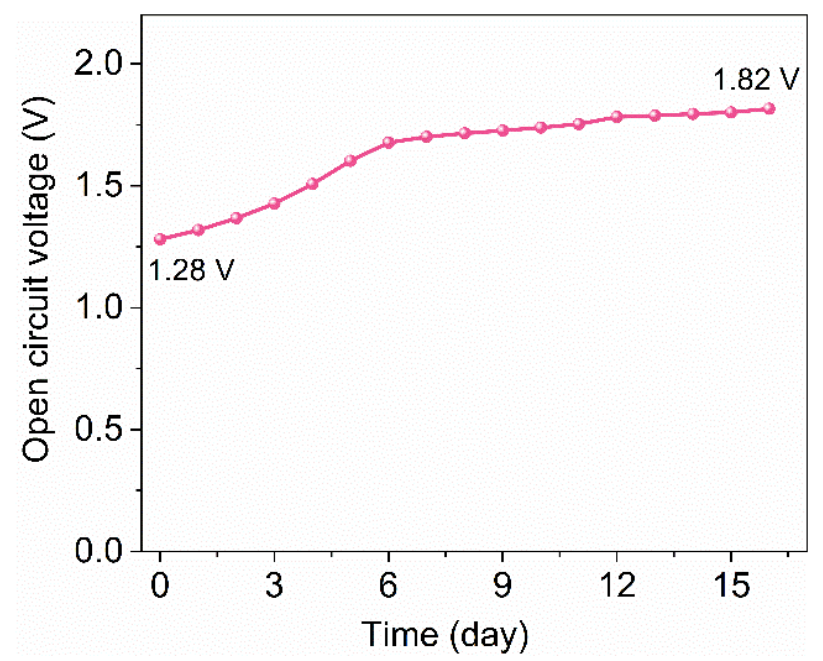

Figure S12. OCV of $\mathrm{Na} / \mathrm{PyDS}$ cells with $\mathrm{Na}-\mathrm{CP}$ electrode as a function of stabilizing time and with $1 \mathrm{M} \mathrm{LiTFSI}$ and $0.15 \mathrm{M} \mathrm{LiNO}_{3}$ as electrolyte. 


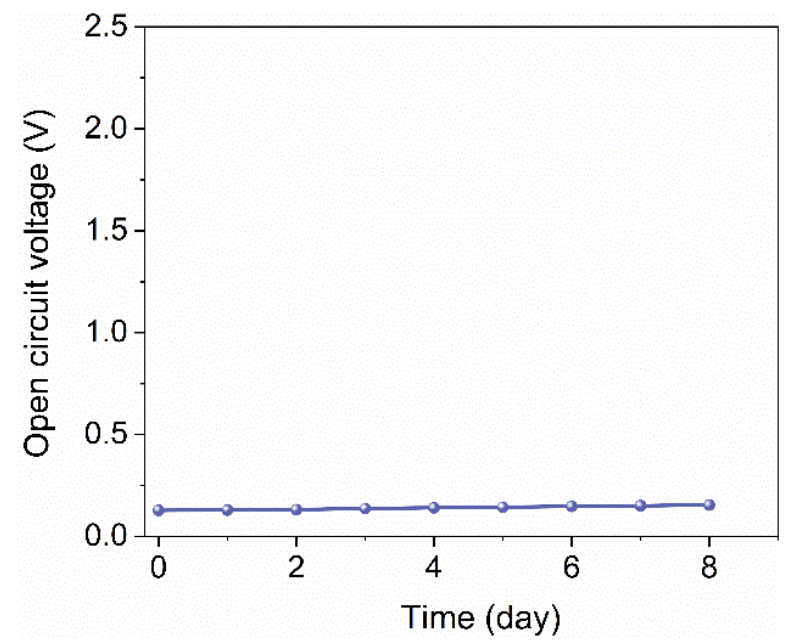

Figure $\mathrm{S}_{13}$. OCV of $\mathrm{Li} / \mathrm{Py}_{2} \mathrm{~S}_{3}$ cells with $\mathrm{Li}-\mathrm{CP}$ electrode as a function of stabilizing time and with $1 \mathrm{M}$ LiTFSI and $0.15 \mathrm{M} \mathrm{LiNO}_{3}$ as electrolyte.
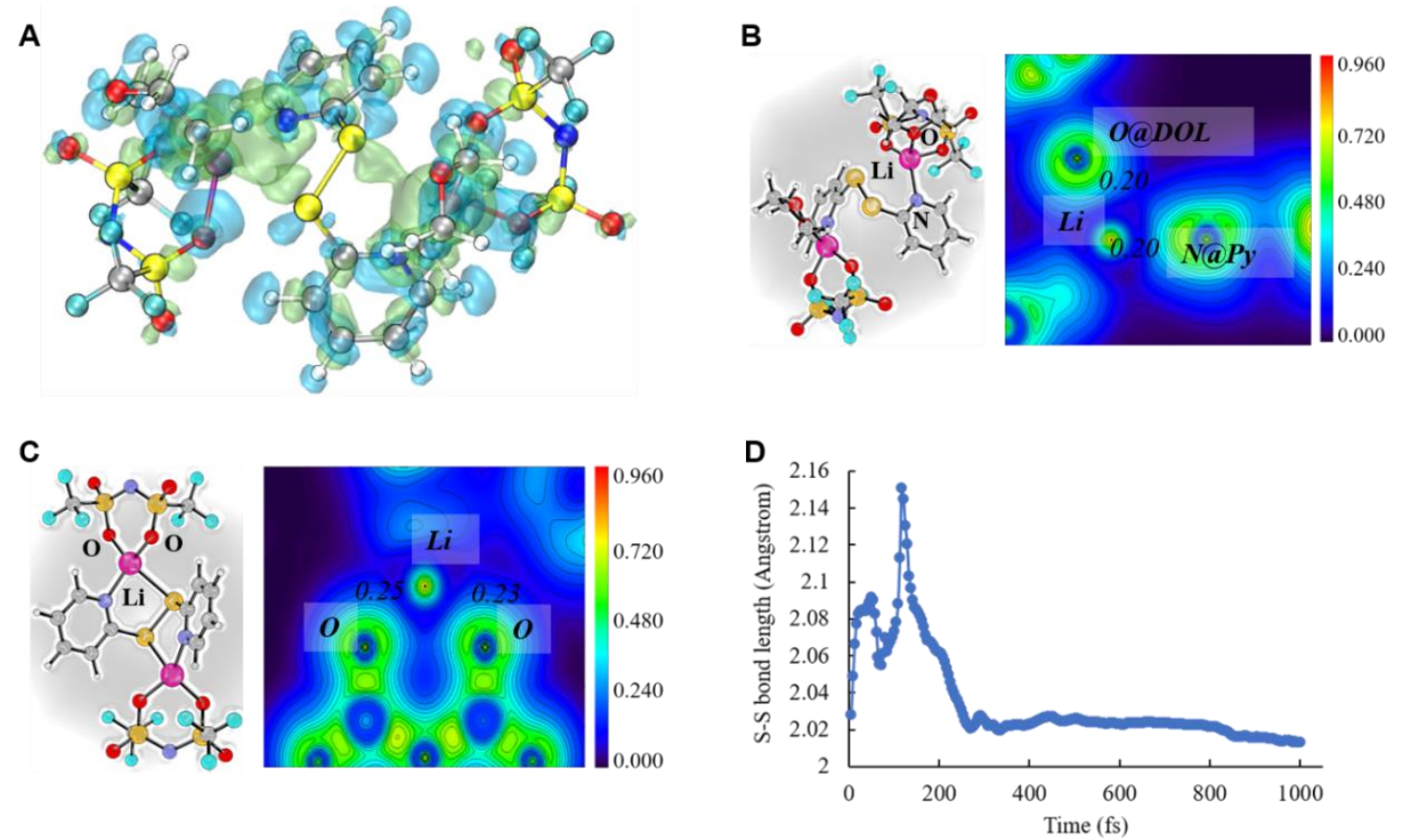

Figure S14. The charge density difference of (A) PyDS-2LiTFSI-2DOL. Green: electronwithdrawing; blue: electron-donating. (B) The optimized geometry (two LiTFSI molecules coordinate with one PyDS) in solvent environment (DOL) and the corresponding twodimensional electron localization function plotting, the bond orders of $\mathrm{Li}-\mathrm{O} @ \mathrm{DOL}$ and $\mathrm{Li}$ N@Py are labeled. (C) The optimized geometry (two LiTFSI molecules coordinate with one PyDS) and the corresponding two-dimensional electron localization function plotting, the bond orders of Li-O are labeled. (D) The S-S bond length of PyDS along the MD simulations of lithium natural atom solvated in the electrolyte. 


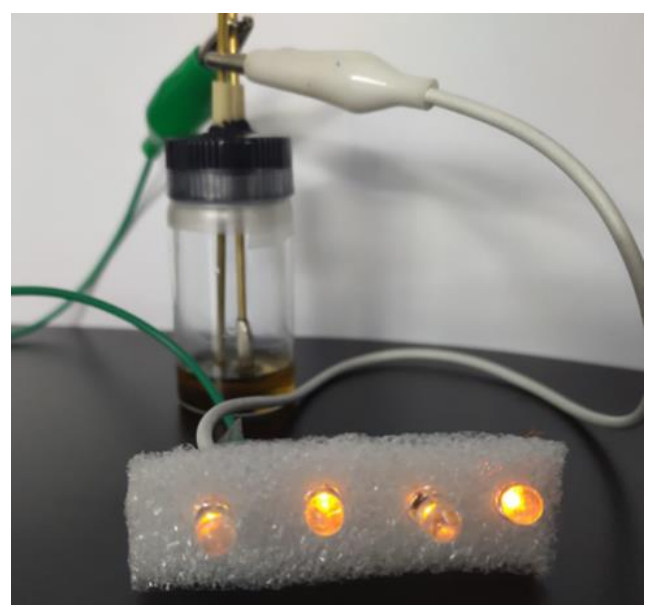

Figure S15. An experimental demonstration of a membrane-free Li/PyDS battery with lithium metal anode and carbon fiber electrode soaking in PyDS catholyte.

A

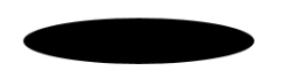

Carbon paper with organosulfide catholytes $(\Phi 12 \mathrm{~mm})$

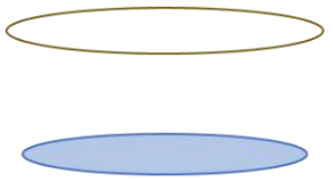

Celgard $2400(\Phi 19 \mathrm{~mm})$

Lithium foil ( $\Phi 15.6 \mathrm{~mm})$

Nickel mesh ( $\Phi 12 \mathrm{~mm})$
B

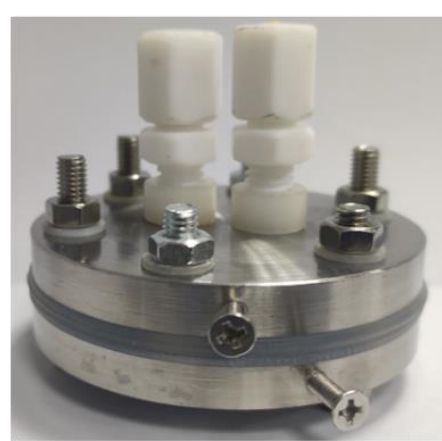

Figure S16. (A) Schematic illustration and (B) photograph of the static Li/organosulfide cell for static test. 


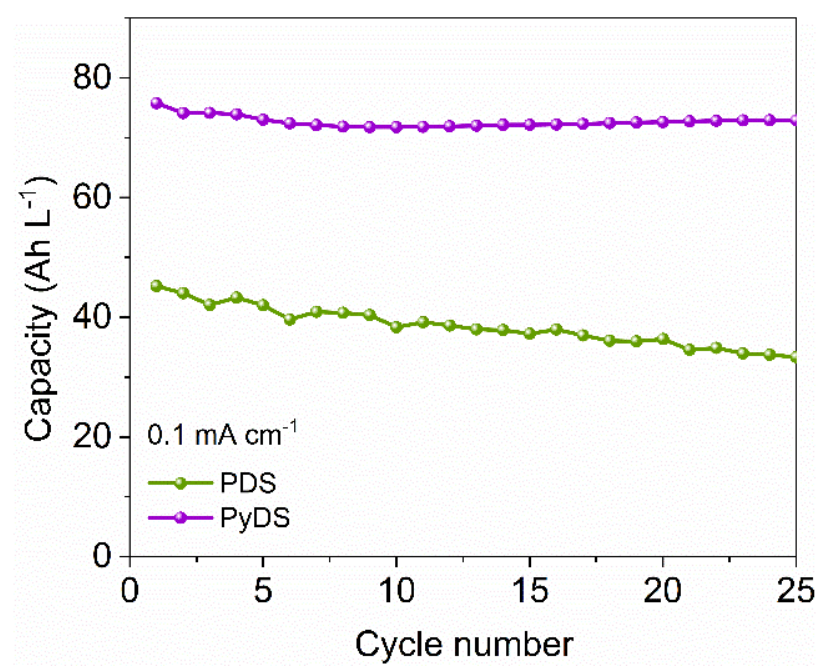

Figure S17. Cycling performance of the Li|PDS and Li|PyDS cells at $0.1 \mathrm{~mA} \mathrm{~cm}{ }^{-2}$.

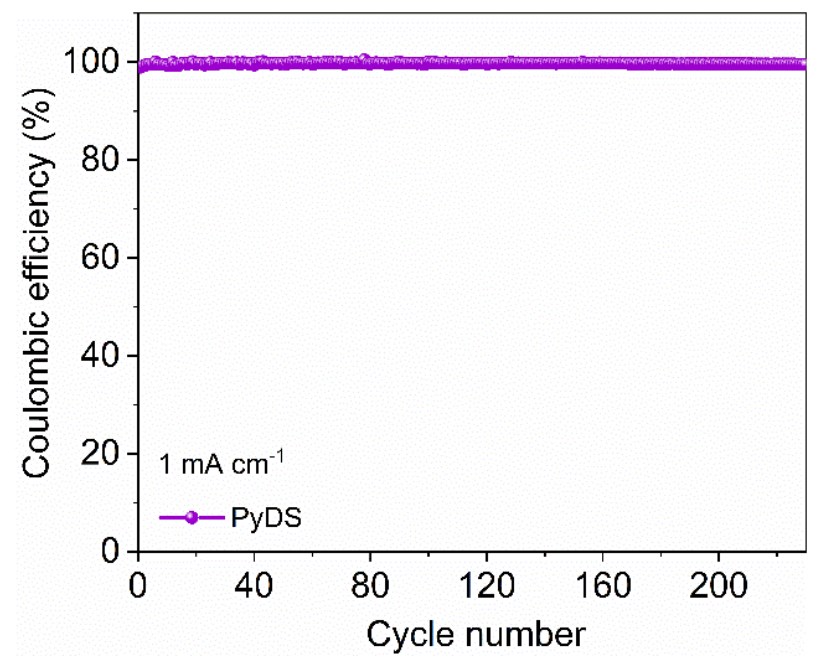

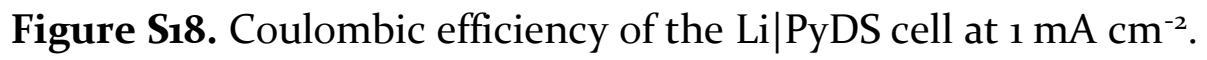



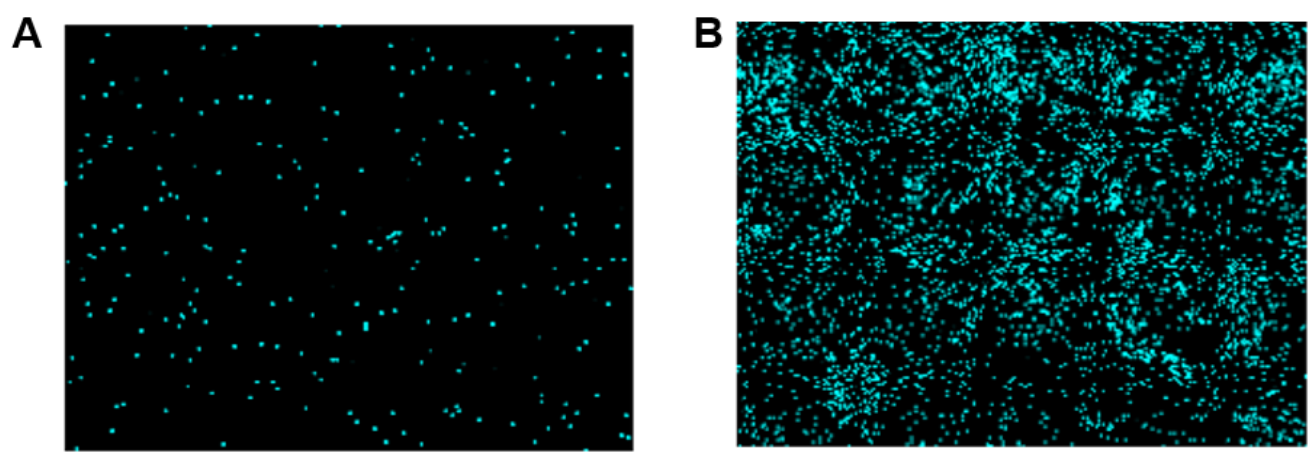

Figure S19. The corresponding sulfur mapping of lithium anode surface paired with (A) PyDS and (B) PDS catholytes after cycling.

Table S1. UV absorption peak areas of PyDS (4.86 min) and PySH (2.39 min) in Li-CP/PyDS electrode in Figure $2 \mathrm{C}$, PDS (7.93 $\mathrm{min})$ and $\mathrm{PhSH}(4.18 \mathrm{~min})$ in $\mathrm{Li}-\mathrm{CP} / \mathrm{PDS}$ electrode in Figure $\mathrm{S}_{5} \mathrm{~A}$.

\begin{tabular}{|c|c|}
\hline Compounds & UV-vis peak area $(\mathbf{m A U} \times \mathbf{m i n})$ \\
\hline PyDS & 316238 \\
\hline PySH & 45678 \\
\hline PDS & 214815 \\
\hline PhSH & 611870 \\
\hline
\end{tabular}

Table S2. UV absorption peak areas of PyDS (4.86 min) and PySH (2.39 min) in Li-CP/PyDS electrode in Figure S8.

\begin{tabular}{|c|c|}
\hline Compounds & UV-vis peak area $(\mathbf{m A U} \times$ min) \\
\hline PyDS & 214833 \\
\hline PySH & 691828 \\
\hline
\end{tabular}


Table S3. The Mulliken charge distribution value of PyDS, PyDS interacting with LiTFSI (denoted as PyDS-(LiTFSI)), LiTFSI, LiTFSI interacting with PyDS (denoted as LiTFSI(PyDS)). " $\Delta$ " represents the Mulliken charge difference between PyDS-(LiTFSI) and the initial PyDS or the Mulliken charge difference between LiTFSI-(PyDS) and initial LiTFSI, and $\Delta \mathrm{M}_{\text {total }}=0.398$ (The total Mulliken charge difference between PyDS-(LiTFSI) and the initial PyDS excluding the Mulliken charge difference of two nitrogen atoms in PyDS); $\Delta \mathrm{M} 2_{\text {total }}=0.140$ (The total Mulliken charge difference between PyDS-(LiTFSI) and the initial PyDS including the Mulliken charge difference of two nitrogen atoms in PyDS); $\Delta \mathrm{M}_{3 \text { total }}=-$ 0.016 (The total Mulliken charge difference between LiTFSI-(PyDS) and the initial LiTFSI excluding the Mulliken charge difference of Li+ in LiTFSI) ; $\Delta \mathrm{M}_{4}$ total $=-0.146$ (The total Mulliken charge difference between LiTFSI-(PyDS) and the initial LiTFSI including the Mulliken charge difference of $\mathrm{Li}^{+}$in LiTFSI). 


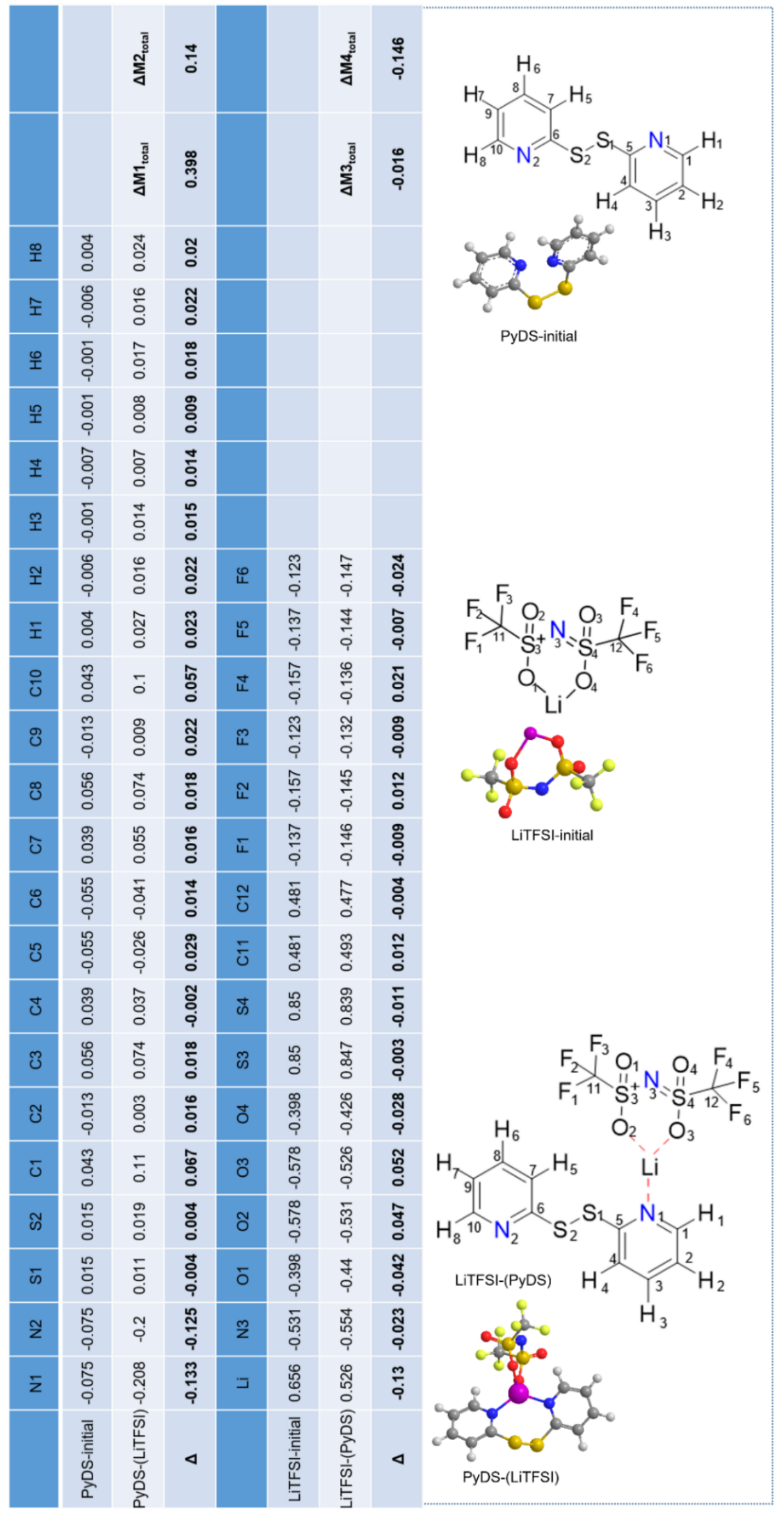




\section{References:}

(1) Fu, Y., Zu, C. \& Manthiram, A. In Situ-formed Li2S in Lithiated Graphite Electrodes for Lithium-Sulfur Batteries. J. Am. Chem. Soc. 2013, 135, 18044-18047.

(2) Kim, H.; Hong, J.; Park, Y.-U.; Kim, J.; Hwang, I.; Kang, K. Sodium Storage Behavior in Natural Graphite using Ether-based Electrolyte Systems. Adv. Funct. Mater. 2015, 25, 534541.

(3) Weigend, F. \& Ahlrichs, R. Balanced basis sets of split valence, triple zeta valence and quadruple zeta valence quality for $\mathrm{H}$ to $\mathrm{Rn}$ : Design and assessment of accuracy. Phys. Chem. Chem. Phys. 2005, 7, 3297-3305.

(4) Gaussian o9, Revision D.o1, M. J. Frisch, G. W. Trucks, H. B. Schlegel, G. E. Scuseria, M. A. Robb, J. R. Cheeseman, G. Scalmani, V. Barone, G. A. Petersson, H. Nakatsuji, X. Li, M. Caricato, A. Marenich, J. Bloino, B. G. Janesko, R. Gomperts, B. Mennucci, H. P. Hratchian, J. V. Ortiz, A. F. Izmaylov, J. L. Sonnenberg, D. Williams-Young, F. Ding, F. Lipparini, F. Egidi, J. Goings, B. Peng, A. Petrone, T. Henderson, D. Ranasinghe, V. G. Zakrzewski, J. Gao, N. Rega, G. Zheng, W. Liang, M. Hada, M. Ehara, K. Toyota, R. Fukuda, J. Hasegawa, M. Ishida, T. Nakajima, Y. Honda, O. Kitao, H. Nakai, T. Vreven, K. Throssell, J. A. Montgomery, Jr., J. E. Peralta, F. Ogliaro, M. Bearpark, J. J. Heyd, E. Brothers, K. N. Kudin, V. N. Staroverov, T. Keith, R. Kobayashi, J. Normand, K. Raghavachari, A. Rendell, J. C. Burant, S. S. Iyengar, J. Tomasi, M. Cossi, J. M. Millam, M. Klene, C. Adamo, R. Cammi, J. W. Ochterski, R. L. Martin, K. Morokuma, O. Farkas, J. B. Foresman, and D. J. Fox, Gaussian, Inc., Wallingford CT, 2013.

(5) Bannwarth, C., Ehlert, S. \& Grimme, S. GFN2-xTB-An Accurate and Broadly Parametrized Self-Consistent Tight-Binding Quantum Chemical Method with Multipole Electrostatics and Density-Dependent Dispersion Contributions. J. Chem. Theory Comput. 2019, 15, 1652-1671.

(6) Humphrey, W., Dalke, A. \& Schulten, K. VMD: Visual Molecular Dynamics. J. Mol. Graphics. 1994, 14, 33-38.

(7) Lu, T. \& Chen, F. Multi wfn: A multifunctional wavefunction analyzer. J. Comput. Chem. 2012, 33, 580-592. 